\title{
Incidencia de los paradigmas didácticos sobre la investigación didáctica y la práctica docente
}

\section{Influence of the didactic paradigms over research in didactics and teaching}

\author{
Josep Gascón, ${ }^{1}$ Pedro Nicolás
}

\begin{abstract}
Resumen: La tesis que tomamos aquí como punto de partida, puede formularse brevemente como sigue: el modelo epistemológico de las matemáticas vigente en una institución didáctica condiciona fuertemente las prácticas docentes que es posible desarrollar en la misma. Uno de los objetivos de este trabajo consiste en clarificar y generalizar esta tesis, mostrando que, más allá del modelo epistemológico subyacente, son los paradigmas didácticos los que permiten explicar las prácticas docentes que la institución lleva a cabo. Paralelamente, al ampliar la noción de teoría didáctica mediante la de praxeología de investigación didáctica, se pone de manifiesto la incidencia del paradigma didáctico que una comunidad científica asume sobre el tipo de problemas de investigación que privilegia y, en última instancia, la importancia del contraste entre paradigmas para profundizar el diálogo entre teorías didácticas.
\end{abstract}

Palabras clave: Paradigma didáctico, praxeología de investigación didáctica, prácticas docentes, fines de la investigación didáctica, fines de la educación matemática.

Fecha de recepción: 29 de mayo de 2020. Fecha de aceptación: 14 de enero de 2021.

${ }^{1}$ Campus de la Universidad Autónoma de Barcelona, Departamento de Matemáticas, Facultad de Ciencias y Biociencias, josepgasconperez@gmail.com, orcid.org/0000-0001-5570-1144.

2 Campus Universitario de Espinardo, Universidad de Murcia, Facultad de Educación, pedronz@um.es, Facultad de Educación 30100 Espinardo (Murcia), orcid.org/0000-0002-6757-9155 


\begin{abstract}
Our starting point is the thesis, perhaps already widely accepted, according to which the epistemological model of mathematics prevailing in a didactic institution strongly affects the teaching in that institution. One of the aims of this work is to enlighten and to generalize this thesis. We will suggest that, rather than just the epistemological model, a complete explanation of teaching practices taking place in a certain institution should also take into account what we call didactic paradigms. At the same time, if we enlarge the notion of theory in didactics by means of the idea of praxeology of research in didactics, it becomes clear that a didactic paradigm is something that can also live or be assumed by such a praxeology of research in didactics, and that affects the kind of research problems considered therein. Therefore, for two theories in didactics to stablish a connection, it is required to make explicit the didactic paradigm assumed by each one.
\end{abstract}

Keywords: Didactic paradigm, praxeology of research in didactics, teaching practices, ends of research in didactics, ends of mathematics education.

\title{
1. INTRODUCCIÓN: HACIA UNA TERCERA ETAPA DEL DIÁLOGO ENTRE TEORÍAS DIDÁCTICAS
}

En 2017, iniciamos un diálogo entre diferentes teorías o enfoques en didáctica de las matemáticas centrado en el presunto carácter normativo de la ciencia didáctica (Gascón y Nicolás, 2017). Más concretamente, esta primera etapa del diálogo ${ }^{3}$ giró en torno a la siguiente cuestión:

¿Hasta qué punto, en qué forma y en qué condiciones, la didáctica puede (o incluso debe) proponer juicios valorativos y normativos que proporcionen criterios sobre cómo organizar y gestionar los procesos de estudio?

Nuestra respuesta, formulada desde el punto de vista de la TAD, fue inequívoca: la ciencia didáctica no está legitimada para enunciar resultados de investigación,

\footnotetext{
${ }^{3}$ Los participantes en esta etapa del diálogo fueron: Guy Brousseau (TSD), Michèle Artigue (contraste entre diversas teorías), Ed Dubinsky (APOS), María Trigueros (APOS), Juan D. Godino (EOS), Koeno Gravemeijer (Educación Matemática Realista), Ricardo Cantoral (TSME) y Josep Gascón y Pedro Nicolás (TAD).
} 
ni juicios de valor, ni prescripciones normativas de ningún tipo (Gascón y Nicolás, 2016/2019a). Esta primera etapa del diálogo dio origen a fuertes debates y acabó provocando la emergencia de nuevas cuestiones y la consiguiente ampliación de la discusión inicial al mostrar la necesidad de sacar a la luz los postulados o asunciones básicas de las diferentes teorías y su relación con los fines que proponen para la investigación didáctica:

¿Cuáles son los fines de la investigación de cada teoría didáctica? En otras palabras, ¿̇cuáles son los problemas de investigación que privilegia y los fenómenos didácticos que se propone explicar? En relación con esto, ¿̇cuáles son los resultados que cada teoría considera admisibles?

En torno a estas cuestiones se ha desarrollado una segunda etapa del diálogo plasmada en múltiples trabajos publicados en For the Learning of Mathematics. ${ }^{4}$ Esta etapa culminó en un curso avanzado presencial organizado por el Centre de Recerca Matemàtica (Barcelona) con ocasión del Intensive Research Program desarrollado entre los días 3 y 14 de junio de 2019.

Entre las cuestiones que han quedado abiertas y que podrían ser tratadas en una tercera etapa del diálogo, Ilamamos la atención sobre las siguientes:

¿Qué postulados fundamentan la investigación didáctica que lleva a cabo una comunidad científica y las prácticas docentes que promueve para alcanzar los fines educativos que, de manera más o menos implícita, propugna dicha comunidad? Y, en última instancia, ¿cómo se relaciona la investigación didáctica que desarrolla una comunidad científica con la práctica docente que promueve?

Veremos, en coherencia con los postulados de la TAD, que para responder a estas cuestiones se requiere, por una parte, integrar la noción de teoría didáctica en la de praxeología de investigación (sección 2) y, sobre todo, profundizar y desarrollar la noción de paradigma didáctico cuyas primeras formulaciones datan de finales del siglo pasado (secciones 3 y ss.). Mostraremos, en definitiva, que los paradigmas que asume de facto una comunidad de investigadores en didáctica inciden simultáneamente sobre su praxis científica y sobre la práctica

\footnotetext{
${ }^{4}$ Hasta el momento se han publicado los siguientes artículos: S. Lerman (2018); J. Proulx (2018); Maria G. Bartolini Bussi (2018); B. Davis (2018) A. Oktaç, M., Trigueros y A. Romo (2019); J. D. Godino, C. Batanero y V. Font (2019); S. Staats y L. A. Laster (2019); J. Gascón y P. Nicolás (2019b).
} 
docente que promueve (sección 8), constituyendo así la piedra de toque para explicar las relaciones entre ambas y para avanzar en el diálogo entre teorías (sección 9).

\section{UN MODELO GENERAL DE LA ACTIVIDAD CIENTÍFICA: LAS PRAXEOLOGÍAS DE INVESTIGACIÓN}

Empezaremos enunciando un postulado antropológico que constituye el núcleo de la teoría de la actividad humana que propone la teoría antropológica de lo didáctico (TAD) en la que nos situamos: toda actividad humana, así como los productos de esta, pueden describirse en términos de praxeologías.

En Chevallard (1999), se describe con todo detalle la estructura y la dinámica de las praxeologías en el caso particular de la actividad matemático-didáctica. Aquí solo diremos que la génesis, personal o institucional, de una praxeología reside en el "bloque de la praxis" $[T / \tau]$ formado por un tipo de tareas $T$ y una técnica $\tau_{T}$ asociada y que su desarrollo requiere de un "bloque tecnológico-teórico" $[\theta / \Theta]$, también Ilamado logos, que está constituido por dos niveles sucesivos, la tecnología $\theta$ y la teoría $\Theta$, de descripción y justificación de la praxis. Utilizamos "tecno-logia" ${ }^{5}$ en un sentido próximo al etimológico, esto es, como discurso razonado (logos) sobre la técnica (tékhne). La noción de "teoría" se interpreta, en primera instancia, como tecnología de la tecnología, esto es, como un discurso razonado de segundo nivel. Es importante subrayar que las cuatro nociones (tipos de tareas, técnicas, tecnologías y teorías) no describen ninguna propiedad intrínseca de los objetos matemáticos, únicamente hacen referencia a las diferentes funciones que estos pueden desempeñar en una actividad. Estas funciones pueden depender de la institución ${ }^{6}$ en la cual se lleva a cabo la actividad (relatividad institucional) e incluso, en una misma institución, un objeto

\footnotetext{
${ }^{5}$ Encontramos un antecedente de este uso en Alexandre Koyré que denominó "tecnología" a la teoría de la práctica y se preguntó por qué la ciencia griega no desarrolló tal tecnología cuya idea, sin embargo, había formulado (Koyré, 1961).

${ }^{6}$ En los trabajos desarrollados en el ámbito de la TAD, la noción de institución ha sido tratada hasta el momento como una noción primitiva. Por su parte, Jesús Mosterín propone la siguiente formulación: Una institución es un conjunto de convenciones o reglas constitutivas que definen y determinan posiciones y relaciones en un entramado social determinado de un modo convencional. Las convenciones o reglas de una institución determinan también derechos y deberes, permisos y prohibiciones, premios y castigos. (Mosterín, 2008, p. 92). Consideramos que esta forma de interpretar las instituciones es compatible con el uso habitual del término en la TAD y que puede servir de punto de partida para precisar dicha noción
} 
matemático puede desempeñar diferentes funciones, dependiendo de la actividad en la que intervenga. La unión, institucional o personal, de un bloque de praxis y un bloque de logos se llama una praxeología y se denota $[T / \tau / \theta / \Theta]$.

En cuanto a la dinámica praxeológica, hay que decir, en primer lugar, que los dos bloques, la praxis y el logos, evolucionan conjuntamente. El bloque del logos condiciona la praxis puesto que acaba determinando las técnicas que están justificadas y su ámbito de validez, así como el tipo de tareas que se pueden abordar con las técnicas disponibles y los resultados que son admisibles. Además, entre las funciones que desempeña el discurso tecnológico figura la construcción de nuevas técnicas y la articulación entre ellas. Recíprocamente, el desarrollo de la praxis va construyendo un corpus de resultados que, a largo plazo, puede integrarse en el bloque del logos enriqueciéndolo mediante nuevas nociones y nuevos discursos tecnológico-teóricos que surgen para describir, justificar e interpretar las sucesivas variaciones de las técnicas.

Cuando este postulado, válido para toda actividad humana, se aplica al caso particular de la actividad científica, se obtiene un modelo general de dicha actividad mediante la noción de praxeología de investigación $\mathrm{PI}=\left[\mathrm{T}_{\mathrm{I}} / \tau_{\mathrm{I}} / \theta_{\mathrm{I}} /\right.$ $\left.\Theta_{\mathrm{I}}\right]$. Esta noción pone de manifiesto que, cuando se habla de teorías científicas para referirse a diferentes enfoques de investigación, se comete una sinécdoque porque se está designando el todo, la praxeología de investigación (PI), por una de sus partes, el componente teórico $\Theta_{\mathrm{I}}$ de la misma. Esta ampliación de la noción de 'teoría científica', el paso de la parte, $\Theta_{\mathrm{I}}$ al todo, $\mathrm{PI}=\left[\mathrm{T}_{\mathrm{I}} / \tau_{\mathrm{I}} / \theta_{\mathrm{I}} / \Theta_{\mathrm{I}}\right]$, es coherente con la evolución actual de la filosofía de la ciencia:

Hasta los años 70 ha imperado una filosofía del conocimiento científico. En las últimas décadas, en cambio, se ha comenzado a desarrollar una filosofía de la actividad científica que, aun siendo complementaria de la epistemología, comienza a interesarse por la práctica de los científicos, no sólo por las teorías científicas. (Echevarría, 1998, p. 7).

La noción de PI constituye, en consecuencia, una ampliación y reconceptualización de la noción de teoría científica que pretende modelizar la actividad científica globalmente considerada y, como tal, constituye una alternativa a los conceptos de paradigma o matriz disciplinar en Kuhn, programa de investigación

(mediante la explicitación de las correspondientes convenciones o reglas constitutivas) en el momento que surjan problemas didácticos que lo requieran. 
en Lakatos, tradición de investigación en Laudan (Diez y Ulises Moulines, 2016) y marco epistémico (Piaget y García, 1981).

En diversos trabajos se ha mostrado que la noción de PI y, en particular, la noción de PI didáctica (PID), es un instrumento útil para describir la estructura y el funcionamiento de la actividad científica, para unificar el lenguaje y para enmarcar el diálogo entre diferentes enfoques de investigación didáctica (Artigue, Bosch y Gascón, 2011a, 2011b; Bosch, Gascón y Trigueros, 2017). ${ }^{7}$

Una PID y, en general toda PI $=\left[T_{\mathrm{I}} / \tau_{\mathrm{I}} / \theta_{\mathrm{I}} / \Theta_{\mathrm{I}}\right]$, está constituida por la unión de una praxis científica $\left[\mathrm{T}_{\mathrm{I}} / \tau_{\mathrm{I}}\right]$ y un logos científico $\left[\theta_{\mathrm{I}} / \Theta_{\mathrm{I}}\right]$. El bloque del logos científico contiene un conjunto de postulados que fijan el tipo de entidades y procesos que forman parte de los componentes de las PID, las relaciones entre ellos y los criterios para describir y justificar la praxis científica. El bloque de la praxis contiene los tipos de problemas científicos que se estudian y las técnicas o metodologías de investigación que se utilizan para estudiarlos. En cuanto a la dinámica de una PID, como en todas las praxeologías, los dos bloques evolucionan conjuntamente. El bloque del logos condiciona la praxis puesto que acabará determinando qué fenómenos didácticos (Artigue, Bosch y Gascón, 2011a) se estudiarán, qué se considerará un problema didáctico y el tipo de resultados de investigación que se aceptarán. En particular, el logos proporciona una heurística positiva (Lakatos, 1981), esto es, un conjunto de orientaciones sobre las líneas de investigación que deben seguirse prioritariamente Recíprocamente, el desarrollo de la praxis de una PID va construyendo un corpus de resultados que, a largo plazo, enriquece la propia metodología de investigación con nuevos métodos de análisis y va añadiendo al bloque tecnológico-teórico nuevas entidades, nuevas relaciones entre ellas y nuevos tipos de fenómenos que hacen evolucionar las asunciones básicas de la PID.

Un ejemplo del enriquecimiento de la metodología de investigación didáctica originado por el desarrollo de la praxis científica lo encontramos, en el caso

\footnotetext{
${ }^{7}$ Con objetivos similares, Luis Radford ha propuesto un espacio conceptual y un metalenguaje para describir las diferentes teorías en Educación Matemática y caracterizar los diferentes tipos de conexiones entre ellas. Así, asocia a cada teoría un trío de componentes (P, M, Q), donde P es un sistema de principios básicos, M una metodología que incluye técnicas de selección e interpretación de "datos" y Q un conjunto de problemas o cuestiones de investigación paradigmáticas (Radford, 2008). Más allá de las posibles relaciones estructurales que, sin duda, pueden establecerse entre $\mathrm{P}, \mathrm{M}$ y $\mathrm{Q}$ por un lado y los componentes de una PID por otro, sería interesante analizar los instrumentos que proporciona cada una de estas ampliaciones de la noción clásica de "teoría científica" para dar cuenta de la práctica de las comunidades de investigación didáctica.
} 
de la TAD, en la emergencia de la noción de modelo epistemológico de referencia (MER). Como consecuencia del desarrollo de dicha noción han surgido nuevas entidades y nuevos tipos de fenómenos que han enriquecido el logos de la TAD, considerada como PID. ${ }^{8}$ En efecto, cuando la teoría de la transposición didáctica (Chevallard, 1985) puso en evidencia la relatividad institucional del saber matemático, la didáctica tuvo que integrarlo como parte de su objeto de estudio y asumir explícitamente la responsabilidad de analizar los modelos epistemológicos del saber matemático que están vigentes en las instituciones que intervienen en los procesos de transposición. Para llevar a cabo dicho análisis es preciso construir modelos epistemológicos específicos o locales, compatibles con un modelo epistemológico general o global, de los diferentes ámbitos de la actividad matemática, a fin de tomarlos como sistemas de referencia útiles para analizar los modelos dominantes o vigentes en las diferentes instituciones:

Toute recherche en didactique qui se propose d'étudier les phénomènes relatifs à un domaine des mathématiques (par exemple l'algèbre élémentaire), et dans une institution didactique donnée, ne devrait pas assumer tel quel le modèle implicite prévalant dans l'institution, mais devrait le prendre en compte en tant qu'objet d'étude, c'est-à-dire comme faisant partie des faits didactiques qui constituent la base "empirique" de la recherche. Pour cela, le chercheur a besoin d'un "point de vue" particulier, c'est-à-dire d'un modèle alternatif du domaine d'activité mathématique enseigné qui lui serve de cadre de référence pour interpréter le modèle dominant dans l'institution qu'il étudie. Or, tout modèle local utilisé pour étudier un domaine particulier des mathématiques enseignées va prendre sa place dans un modèle global de l'activité mathématique qui restera, selon les cas, plus ou moins explicité par le chercheur. (Gascón, 1994-95, p. 44)

A los citados "modelos alternativos", que presentan un carácter relativo y provisional, y cuya construcción es responsabilidad de la didáctica, se les asignó desde el principio una función fenomenotécnica:

\footnotetext{
${ }^{8}$ Si bien es cierto que, como afirma uno de los revisores de este trabajo, "les praxéologies de recherche que nourrit la $T A D$, héritières de cette histoire, sont bien plus diverses dans leurs problématiques et leurs méthodologies", en este trabajo identificamos la TAD con una PID relativamente bien definida cuyo logos y cuya praxis científica hemos intentado caracterizar en (Gascón y Nicolás, 2016/2019a y 2019b).
} 
[...] en la interpretación -e incluso en la mera formulación- de los fenómenos didácticos, [...] es imprescindible la utilización de un modelo específico de la forma como se generan y se desarrollan los conocimientos matemáticos involucrados en dichos fenómenos. (Gascón 1993, p. 302)

Toda PID asume en el logos y utiliza en la praxis, de manera más o menos explícita, un modelo epistemológico de los conocimientos en juego. De hecho, en (Gascón, 2003) hemos caracterizado las PID que se sitúan en el Programa Epistemológico de Investigación Didáctica como aquellas que explicitan los MER que inevitablemente utilizan para formular los problemas didácticos que abordan.

This is precisely the originality of the Epistemological Program in the didactics of mathematics. It opens a new way of access to the study of the didactic phenomena through the explicit modelization of the taught mathematical knowledge (Gascón, 2003, p. 50).

Es precisamente esta toma de conciencia de los MER que emplea una comunidad didáctica lo que permite: (a) utilizarlos como hipótesis provisionales en lugar de considerarlos como postulados inamovibles; y (b) disponer de un sistema de referencia para contrastarlo con el modelo epistemológico vigente (MEV) ${ }^{9}$ en cada una de las instituciones que forman parte de su objeto de estudio. Así, IOS MER se constituyen como instrumentos de emancipación epistemológica de la comunidad didáctica (Gascón, 2014).

Junto a la asunción de un modelo epistemológico concreto de los conocimientos en juego, el logos de una PID tiende a privilegiar ciertos fines educativos que se formulan con las nociones y los términos que proporciona dicho modelo, al tiempo que propone unos medios didácticos, entendidos como prácticas docentes o estrategias didácticas, supuestamente útiles para alcanzar dichos fines. En consecuencia, en su praxis científica, la comunidad de investigadores que trabaja en el ámbito de una PID suele formular problemas de investigación utilizando los términos que proporciona el modelo epistemológico subyacente y haciendo referencia a los citados fines y medios. Es en este punto en el que surge la noción de paradigma didáctico que analizaremos con detalle en las próximas secciones.

\footnotetext{
${ }^{9}$ En trabajos anteriores hemos denominado modelo epistemológico dominante (MED) al que aquí denominamos modelo epistemológico vigente (MEV) en una institución docente.
} 


\section{ORIGEN Y DESARROLLO DE LA NOCIÓN DE PARADIGMA DIDÁCTICO}

Las primeras formulaciones de la noción de paradigma didáctico datan de (Gascón, 1992, 1994). Posteriormente, la noción de paradigma didáctico se fundamentó epistemológicamente mediante una reconstrucción racional, en el sentido de (Lakatos, 1971), de la evolución histórica del problema epistemológico (de las matemáticas) y la caracterización de tres tipos de modelos epistemológicos: euclideanistas, cuasiempiristas y constructivistas que constituyen esquematizaciones de las respuestas históricas a las sucesivas formulaciones de dicho problema. Cada uno de estos tipos de modelos epistemológicos sustenta un tipo de "modelos docentes" o "paradigmas (didácticos) ideales". En particular, el paradigma teoricista se sustenta en una epistemología euclideanista; el paradigma modernista en una epistemología cuasi empirista, y el paradigma de la modelización matemática en cierta versión de la epistemología constructivista (Gascón, 2001).

Los paradigmas didácticos y los modelos epistemológicos citados son construcciones teóricas, formas ideales, que nunca han existido en estado puro en alguna institución. Unos y otros tienen una función metodológica, heurística, se utilizan para ser contrastados con la realidad empírica. Sus rasgos definitorios pueden estar o no presentes en una institución docente (en un periodo histórico concreto) pero los paradigmas y los modelos epistemológicos vigentes efectivamente en la contingencia institucional, presentan siempre un carácter mixto formado por rasgos de las diferentes formas ideales, son mestizos.

La tesis de (Gascón, 2001), que tomamos aquí como punto de partida, puede formularse brevemente como sigue: el modelo epistemológico de las matemáticas dominante (o vigente) en una institución didáctica condiciona fuertemente las prácticas docentes que es posible desarrollar en la misma. Uno de los objetivos de este trabajo consiste en clarificar y generalizar la tesis anterior mostrando que, más allá del modelo epistemológico subyacente, son los paradigmas didácticos globalmente considerados los que permiten explicar las prácticas docentes -las acciones didácticas- que la institución lleva a cabo o que una PID propugna, así como el tipo de problemas didácticos que la institución considera relevantes o que una PID privilegia como problemas de investigación didáctica. 


\section{PARADIGMA DIDÁCTICO VIGENTE EN UNA INSTITUCIÓN Y PARADIGMAS DIDÁCTICOS DE REFERENCIA CONSTRUIDOS POR UNA PRAXEOLOGÍA DE INVESTIGACIÓN DIDÁCTICA}

La nueva versión que proponemos de la noción de paradigma didáctico fue presentada en Gascón y Nicolás (2018), y depende esencialmente: (a) de los fines educativos que propugna; y (b) del modelo epistemológico en el que se sustenta.

(a) En cuanto a los fines educativos, ${ }_{10}^{10}$ hemos de distinguir entre los fines que, de manera más o menos explícita, persigue una institución docente, que podríamos denominar fines educativos vigentes $\left(F_{V}\right)$ en dicha institución, y los fines que una PID (como, por ejemplo, la TAD) utiliza como fines educativos de referencia $\left(F_{R}\right)$. Unos y otros se sitúan en la "esfera de los valores" (Weber, 1917/2010), caen fuera de la "esfera del conocimiento" y, en consecuencia, no pueden ser establecidos racionalmente.

Los $F_{V}$ en una institución escolar quedan plasmados parcialmente en los documentos curriculares y pueden rastrearse en múltiples expresiones sociales y culturales formando parte de lo que Isaiah Berlín denominaba "ideas generales" de una sociedad, esto es, creencias, actitudes, hábitos y disposiciones individuales y sociales que en el lenguaje común (y a causa del legado marxista) solemos Ilamar ideología (Berlín, 2017). La ideología pedagógica de la sociedad se establece en coherencia con su estructura social y ambas evolucionan a lo largo de la historia de las sociedades en función de las normas que las rigen en cada periodo histórico (Durkheim, 1924/1991). Por su parte, los $F_{R}$ que una PID utiliza, pueden ser asumidos por esta, tomándolos como postulados relativamente incuestionables, o utilizados como una hipótesis provisional, un sistema de referencia, para analizar la realidad empírica.

(b) En lo que se refiere a los modelos epistemológicos en los que se sustentan los paradigmas didácticos, hemos de distinguir entre el modelo epistemológico vigente (MEV) en una institución docente, en un periodo histórico determinado, y los modelos teóricos que construye una PID para analizar y evaluar, entre otras cosas, los citados MEV, y que denominamos modelos epistemológicos de referencia (MER). En coherencia con los postulados de la TAD,

\footnotetext{
${ }^{10}$ Hay que distinguir, asimismo, entre los fines últimos y los fines intermedios. Estos son medios para alcanzar los fines últimos y, en este sentido, "los fines intermedios son justificables en función de los fines últimos" (Mosterín, 2008, p. 35).
} 
los modelos epistemológicos de las matemáticas (ya sean modelos generales o modelos específicos de los diferentes dominios de estas) pueden describirse en términos praxeológicos. Los MER que construye la TAD pueden representarse mediante relaciones relativamente bien definidas (por ejemplo, relaciones de inclusión o relaciones de modelización) entre praxeologías matemáticas cuyos componentes están claramente explicitados. Mientras que, por su parte, el MEV en una institución suele contener relaciones relativamente imprecisas entre praxeologías matemáticas que presentan incompletitudes e incoherencias que aparecen cuando se analizan desde el punto de vista que proporciona un MER.

En torno a los fines educativos que están vigentes $\left(F_{v}\right)$ y son compartidos en una institución y al modelo epistemológico vigente (MEV) en esta, se constituye un proyecto educativo que lleva asociado una forma específica de analizar, describir y evaluar la educación matemática escolar, esto es, lo que denominamos el paradigma didáctico vigente (PDV) en dicha institución y que incluye, además, los medios $\left(M_{v}\right)$ supuestamente útiles para alcanzar esos fines. Apelando de nuevo al postulado de la TAD según el cual toda actividad humana puede describirse en términos de praxeologías, diremos que los medios que un paradigma propone son procesos didácticos (o procesos de estudio) de cierto tipo que se pueden describir en términos de praxeologías que denominamos praxeologías didácticas (Chevallard, 1999).

En general, los paradigmas didácticos -tanto si son construcciones culturales de una institución educativa como si están creados por una PID para utilizarlos como paradigmas de referencia- no surgen de la nada. Se constituyen como reacción a un estado de cosas (real o hipotético) previamente establecido, esto es, como respuesta a un paradigma vigente o simplemente posible. En consecuencia, para caracterizar un paradigma didáctico necesitamos, también, explicitar los hechos didácticos a los que pretende responder. Con más precisión: un paradigma didáctico permite interpretar como fenómenos didácticos ciertas "regularidades" observables en los procesos didácticos que tienen lugar en la institución docente en la que está vigente (real o hipotéticamente) otro paradigma. Podríamos resumir lo anterior diciendo que un paradigma didáctico es uno de los instrumentos que utiliza el didacta para sacar a la luz y hacer visibles ciertos hechos didácticos (ignorando otros) y para proporcionar una interpretación de estos, como fenómenos didácticos. Esta interpretación se basa habitualmente en que el nuevo paradigma permite relacionar entre sí hechos que eran considerados independientes según las lentes del paradigma vigente. 
En definitiva, para caracterizar el PDV en una institución en cierto periodo histórico, hemos de explicitar: el MEV en dicha institución; los $F_{V}$ que dicho paradigma propugna; los $M_{v}$ que se consideran necesarios en la institución para alcanzar dichos fines y que se materializan en determinadas praxeologías didácticas; y los fenómenos ( $\varphi v$ ) que el paradigma saca a la luz y a los que responde. En síntesis, caracterizaremos el PDV en una institución mediante cuatro componentes interrelacionados:

$$
\mathrm{PDV}=[\mathrm{MEV}, \mathrm{Fv}, \mathrm{Mv}, \varphi \mathrm{v}]
$$

Análogamente, caracterizaremos los paradigmas didácticos que construye una PID (como, por ejemplo, la TAD) y que denominaremos paradigmas didácticos de referencia (PDR), mediante cuatro componentes:

\section{$P D R=\left[M E R, F_{R}, M_{R}, \varphi R\right]$}

EI MER constituye una forma concreta de redefinir o reinterpretar cierto dominio de las matemáticas (o, en su caso, las matemáticas globalmente consideradas); $F_{R}$ se refiere a los fines educativos que dicho paradigma propugna; $M_{R}$ designa los medios que se consideran necesarios para alcanzar los fines perseguidos; $y$ $\varphi_{R}$ los fenómenos didácticos que el MER permite sacar a la luz y a los que el paradigma en cuestión pretende responder.

En consecuencia, Ios PDR construidos por una PID pueden considerarse instrumentos de emancipación de la didáctica respecto de múltiples sujeciones institucionales: respecto a las formas como las instituciones docentes describen y estructuran el conocimiento matemático (emancipación epistemológica); respecto de los fines de la educación matemática compartidos en dichas instituciones; respecto de los medios que se suponen útiles para alcanzar dichos fines; y, también, respecto de la selección de los hechos didácticos que son visibles institucionalmente y de la forma de interpretarlos. En definitiva, Ios PDR son instrumentos de emancipación de la didáctica respecto del PDV en las instituciones que forman parte de su objeto de estudio, ampliando así el papel emancipatorio que desempeñan los MER (Gascón, 2014).

De hecho, Ios PDR explicitan y precisan el contenido de la hipótesis que formula el MER en el que se sustentan. En efecto, recordemos que un MER es una hipótesis científica -no una mera reformulación o descripción alternativa de cierto dominio de las matemáticas- y, como tal, es provisional y debe ser 
contrastado empíricamente. Un MER en torno a cierto dominio de la matemática escolar comporta habitualmente la hipótesis de que la nueva razón de ser que este asignará a dicho dominio permitirá superar las "limitaciones" de las organizaciones didácticas escolares vigentes en torno al mismo y, simultáneamente, que el MER cumplirá su función fenomenotécnica sacando a la luz ciertos fenómenos que eran invisibles desde la mirada que proporcionaba el MEV en dicha institución.

En resumen, un $\mathrm{PDR}=\left[\mathrm{MER}, \mathrm{F}_{\mathrm{R}}, \mathrm{M}_{\mathrm{R}}, \varphi_{\mathrm{R}}\right]$ construido como respuesta al paradigma vigente, $\mathrm{PDV}=[\mathrm{MEV}, \mathrm{Fv}, \mathrm{Mv}, \varphi \mathrm{v}]$, amplía y precisa la hipótesis científica que propone el MER, puesto que, además de explicitar una forma concreta de reformular el MEV (mediante el MER), marca una posible dirección de cambio de la práctica docente (la indicada por los fines educativos $F_{R}$ ) y unos medios $M_{R}$ presuntamente útiles para alcanzar dichos fines.

En definitiva, las nociones de PDR y PDV generalizan respectivamente y completan las antiguas nociones de MER y de MEV. Se pone así de manifiesto que ha sido necesario explicitar el papel de los fines educativos asociados al modelo epistemológico en cuestión y subrayar la importancia de los fenómenos didácticos que este permite sacar a la luz, para construir la noción de paradigma didáctico. ${ }^{11}$

${ }^{11}$ Corine Castela denomina paradigma de investigación al compuesto formado por una praxeología de investigación, PI, y una organización social correlacionada, una comunidad de investigadores, que funciona como una institución que da existencia a la PI y que permite que esta sea reconocida en el ámbito de la enseñanza de las matemáticas (Castela, 2015). Como es evidente, esta noción (que la propia autora considera que puede ser "provisional") tiene poco que ver con nuestra noción de paradigma didáctico. Para empezar, nuestra interpretación de las praxeologías (y, en particular, de las PI) presupone la existencia de una institución en la que estas viven y cuyos sujetos son actores que, en mayor o menor medida y con mayor o menor éxito, abordan ciertos tipos de tareas con algunas de las técnicas que están disponibles en la institución. Son estos sujetos-actores los que, colectivamente, construyen discursos (o los toman de la institución) que pretenden describir, interpretar, desarrollar y justificar su praxis. En consecuencia, no consideramos que sea necesario completar una PID añadiéndole una comunidad de investigadores porque no hay praxis ni logos sin actores y toda actividad humana es una actividad institucionalizada. Además, en lo que sigue, mostraremos que los paradigmas didácticos asumidos de facto por una comunidad de investigadores inciden simultáneamente sobre su praxis cientifica (haciendo evolucionar el logos de dicha PID y modulando así el PID que dicha comunidad comparte) y sobre la práctica docente que dicha comunidad promueve. 


\section{PARADIGMAS DIDÁCTICOS SITUADOS EN LOS DIFERENTES NIVELES DE CODETERMINACIÓN}

Hasta aquí, al referirnos a los componentes de un paradigma didáctico, hemos hablado de "fines educativos" en un sentido genérico, de "fines de la educación matemática" e, incluso, de "modelos epistemológicos de cierto dominio matemático". Para precisar el nivel de generalidad en el que nos situamos, hemos generalizado la noción de paradigma didáctico para modelizar y ordenar conceptualmente la realidad empírica educativa no sólo en el nivel disciplinar (matemático) sino también en el pedagógico (independiente de las disciplinas escolares) y en los niveles subdisciplinares. En consecuencia, junto a algunos paradigmas disciplinares (relativos a las matemáticas globalmente consideradas), caracterizaremos algunos pedagógicos y aludiremos a ciertos paradigmas subdisciplinares, esto es, paradigmas didácticos que se sitúan en el nivel de los diferentes ámbitos de la actividad matemática escolar.

En todos los niveles, los PDR permiten modelizar, y en consecuencia estudiar, los proyectos educativos históricamente existentes o posibles. Sirven como herramientas heurísticas para analizar y evaluar la forma como se conceptualiza, en una institución determinada, la educación escolar en cada uno de dichos niveles. De hecho, una forma de describir y analizar el PDV en una institución consiste en compararlo con ciertos PDR que revelarán, por analogía o por contraste, algunos rasgos del PDV en cuestión.

Distinguir entre paradigmas situados en los diferentes niveles de codeterminación didáctica (Chevallard, 2002) nos permite diferenciar claramente entre modelos epistemológicos, fines educativos, medios y, también, entre fenómenos didácticos que se sitúan ya sea en el nivel pedagógico, disciplinar (matemático), o en los diferentes niveles subdisciplinares. Es muy importante subrayar que los fenómenos didácticos emergentes en los niveles subdisciplinares como, por ejemplo: el aislamiento de la proporcionalidad (García, Gascón, Ruiz Higueras y Bosch, 2006); la identificación del álgebra elemental con la aritmética generalizada (Bolea, 2002); la desarticulación entre el álgebra elemental y la modelización funcional (Ruiz-Munzón, 2010); la reducción de la razón de ser de los sistemas de numeración a la mera designación de los números (Sierra, 2006); la ausencia escolar de una razón de ser del cálculo diferencial elemental que sea coherente con el papel que desempeña en la actividad científica (Lucas, 2015); o la desarticulación entre los números y la medida de magnitudes (Licera, 2017), no pueden ser explicados utilizando únicamente un modelo 
epistemológico general de las matemáticas ni, mucho menos, si sólo se dispone de un modelo genérico de los conocimientos en juego. Los trabajos citados muestran que se requiere, en cada caso, utilizar un modelo epistemológico específico del ámbito matemático que está en juego.

En lo que sigue, describiremos concisamente algunos PDR disciplinares (matemáticos) construidos en (Gascón, 2001) y, a continuación, mostraremos su relación con dos PDR pedagógicos construidos recientemente por la TAD para contrastarlos con los PDV (u otros posibles) en las instituciones escolares.

\section{PARADIGMAS DIDÁCTICOS DISCIPLINARES DE REFERENCIA}

Mostraremos con tres ejemplos en lo que sigue, que los fines educativos que un paradigma didáctico propugna están condicionados por el modelo epistemológico en el que se sustenta dicho paradigma. Estos fines influyen, a su vez, sobre el tipo de prácticas docentes (los medios) que es coherente llevar a cabo en dicho paradigma y delimitan los hechos didácticos visibles en los correspondientes procesos de estudio, así como su interpretación como fenómenos. En consecuencia, los componentes de un paradigma didáctico, especialmente si nos referimos a un PDR construido por una PID, presentan cierta unidad funcional, por lo que deben analizarse conjuntamente, como un sistema complejo. Por lo tanto, la construcción de PDR y el estudio de los PDV (o posibles) constituyen actividades esenciales en la metodología de la TAD. ${ }^{12}$

Para avanzar en esa dirección, los primeros PDR fueron construidos en (Gascón 2001), encontrándose antecedentes en (Gascón, 1992, 1994). En estos trabajos, para precisar y ejemplificar la relación entre algunos modelos epistemológicos (generales) de las matemáticas y los fines de la educación matemática asociados solidariamente con cada uno de ellos, se definían diferentes PDR disciplinares. Cada PDR se caracterizaba, en primer lugar, mediante el modelo epistemológico subyacente y, complementariamente, aludiendo a los

${ }^{12}$ Forma parte de dicha metodología la cuestión de evaluar la coherencia interna de un paradigma didáctico, esto es: ¿̇los medios que propone son útiles para alcanzar los fines que propugna?, ¿̇el modelo epistemológico que sustenta el paradigma permite sacar a la luz los fenómenos a los que este pretende responder?, ¿cómo se utilizan las nociones que proporciona el modelo epistemológico para formular los fines?, etc. También forma parte de la metodología de la TAD la evaluación de la coherencia externa, esto es, la coherencia entre un paradigma disciplinar y el paradigma pedagógico del que depende o entre un paradigma disciplinar y los paradigmas subdisciplinares que dependen de él. 
fines educativos que perseguía y a los medios didácticos que proponía para alcanzar dichos fines. En todos los casos, los paradigmas definidos en dichos trabajos (teoricismo, tecnicismo, modernismo, modelizacionismo, etc.) eran propuestos como tipos ideales que, como tales, no han existido nunca en estado puro en alguna institución. Cada uno de ellos caracterizaba una forma hipotética de describir, analizar y evaluar la educación matemática globalmente considerada.

Cada uno de estos PDR respondía al estado de cosas originado por la vigencia (histórica o hipotética) de otro paradigma, cuyas "limitaciones" el nuevo paradigma pretendía "superar" en la dirección marcada por los "nuevos" fines que el PDR en cuestión propugnaba. En consecuencia, Ios PDR disciplinares construidos en (Gascón, 2001) ya estaban estructurados, al menos implícitamente, mediante los cuatro componentes descritos: PDR $=\left[M E R, F_{R}, M_{R}, \varphi_{R}\right]$ que conviene explicitar en cada caso en aras de clarificar las cosas.

En esta sección describiremos muy brevemente, a título de ejemplo, tres de estos PDR disciplinares que fueron definidos basándonos en la teoría de los momentos didácticos (Chevallard, 1999, pp. 250-255). Los dos primeros ejemplos (teoricismo y modernismo) constituyen PDR unidimensionales porque cada uno de ellos enfatiza una única dimensión de la actividad matemática (en el sentido de la citada teoría de los momentos). El tercero, el modelizacionismo, constituye una completación relativa tanto del teoricismo como del modernismo. Mostraremos, además, que el paradigma teoricista y el de la modelización matemática, pueden considerarse como el reflejo, a nivel disciplinar, de sendos PDR que se sitúan en el nivel pedagógico y que han sido construidos recientemente (Chevallard, 2013a, 2013b).

\subsection{El PARADIGMA TEORICISTA: ENSEÑAR MATEMÁtICAS ES "MOSTRAR" TEORÍAS CRISTALIZADAS}

El teoricismo es un PDR a nivel disciplinar matemático cuyo modelo epistemológico subyacente es el euclideanismo (Lakatos, 1978) que desempeña, por tanto, el papel de MER de este paradigma disciplinar. Este modelo epistemológico general de las matemáticas identifica el conocimiento matemático con las teorías cristalizadas, centrando la actividad matemática en la que se lleva a cabo en el momento de la constitución del entorno tecnológico-teórico. En este paradigma los argumentos deductivos desempeñan un papel esencial, lo que 
comporta la necesidad de llevar a cabo un análisis lógico de las nociones que se utilizan y el compromiso de expresarlas en el lenguaje de la lógica de primer orden.

Por razones de economía didáctica el euclideanismo traslada el momento tecnológico-teórico para hacerlo coincidir con el momento del primer encuentro (Chevallard, 1999) que se sitúa en la primera etapa del estudio, relegando la resolución de problemas a simples aplicaciones del bloque tecnológico-teórico. En la medida en que se impone esta postura epistemológica, los restantes momentos del proceso didáctico quedan relegados a un segundo plano y aparecen muy condicionados por el momento dominante. Así, por ejemplo, los momentos de la evaluación, tan importantes en todo proceso de estudio, quedan restringidos a evaluar los fines que propugna el paradigma teoricista sustentado en el euclideanismo.

En coherencia con dicho modelo epistemológico, el teoricismo considera que el proceso didáctico empieza, y prácticamente acaba, cuando el profesor "enseña" (en el sentido de "muestra") las teorías cristalizadas a los alumnos. En este paradigma los fines de la educación matemática $\left(F_{R}\right)$ se centran en "enseñar" a los estudiantes teorías matemáticas perfectamente acabadas y cristalizadas. Los medios $\left(M_{R}\right)$ que se proponen para alcanzar estos fines se reducen a una presentación "autoritaria" de ciertas teorías matemáticas incuestionables y a poner en marcha actividades, consideradas como subsidiarias, para aplicar, ejemplificar y consolidar los conceptos y teoremas que estructuran dichas teorías y, también, para motivarlas, introducirlas o justificarlas, siempre con la finalidad de que el alumno adquiera un cuerpo de conocimientos predeterminado de antemano. Se produce así una cierta trivialización de la actividad matemática que proviene del dogma euclidiano según el cual todos los conocimientos que el alumno necesitará emplear en su práctica matemática están contenidos en la teoría matemática. Se supone que en esta (tal como la describe el estilo euclideanista) están contenidos esencialmente todos los conocimientos que se necesitan. Se considera que para llevar a cabo cualquier actividad matemática basta elegir cuál es el teorema adecuado o la definición pertinente en cada caso, puesto que una vez que se ha dado con ellos, el resto de la actividad es prácticamente trivial (Gascón, 2001). Es obvio que el euclideanismo como modelo epistemológico de las matemáticas y los fines de la educación matemática que propugna el paradigma teoricista se condicionan mutuamente.

Para describir los fenómenos a los que responde el paradigma teoricista $\left(\varphi_{R}\right)$ debemos aludir a una situación que se produjo en el seno de la comunidad 
matemática, algo lejana en el tiempo, pero que todavía resuena en algunas instituciones universitarias. Nos referimos a la existencia de contradicciones y paradojas alarmantes que aparecieron a finales del siglo XIX y principios del siglo XX que hicieron tambalear los fundamentos de las matemáticas y provocaron la necesidad de buscar bases sólidas para fundamentarlas lógicamente (Lakatos, 1981).

En la medida en que el paradigma teoricista predomina en una institución, se originan fenómenos didácticos entre los que hay que destacar aquellos que están relacionados con la citada trivialización de la actividad matemática y con la estrategia didáctica asociada que se materializa en la presentación autoritaria de las obras mediante un estilo deductivista: "Aún no se ha constatado suficientemente que la educación matemática y científica actual es un semillero de autoritarismo, siendo el peor enemigo del pensamiento crítico e independiente" (Lakatos, 1978, p. 166).

Esta estrategia tiende a silenciar todo tipo de preguntas de los estudiantes, con la consiguiente reducción de su papel al de simples ejecutantes con poca autonomía y, en los casos límite, al de meros espectadores. Es por esta razón que Lakatos asociaba el autoritarismo de la educación matemática con el euclideanismo e indicaba que, de acuerdo con el ideal euclídeo, el estudiante se ve obligado a asistir a una especie de ceremonia sin hacer preguntas sobre el trasfondo problemático que está tras los argumentos.

\subsection{EL PARADIGMA MODERNISTA: APRENDER MATEMÁTICAS MEDIANTE UNA EXPLORACIÓN LIBRE}

Desde el punto de vista que proporciona la TAD, podemos afirmar que las formas extremas del paradigma teoricista presentan limitaciones evidentes en la gestión del proceso de estudio de las matemáticas. En particular el postulado teoricista según el cual todo lo necesario para "hacer matemáticas" está contenido en la teoría, provoca una situación insostenible en las instituciones en las que todavía predomina dicho paradigma. En efecto, Ios alumnos tropiezan, en la práctica, con enormes dificultades cuando intentan construir de manera autónoma técnicas matemáticas eficaces sustentándose únicamente en sus conocimientos teóricos. 
El paradigma modernista (Gascón, 2001) constituye una reacción ante estos hechos y ante las consecuencias del paradigma tecnicista. ${ }^{13}$ La respuesta del modernismo a los citados fenómenos $\left(\varphi_{R}\right)$ se materializa tomando la exploración libre y creativa de cuestiones abiertas como los principales fines de la educación matemática $\left(F_{R}\right)$. Se enfatiza así el momento exploratorio (Chevallard, 1999), esto es, la exploración de ciertos tipos de tareas y la consiguiente indagación en la búsqueda de "materiales" que permitan elaborar una técnica útil para abordar dichas tareas. El modernismo se centra en el momento exploratorio, situando en segundo plano los restantes momentos del proceso didáctico que aparecen condicionados y restringidos por el momento dominante. Al igual que el teoricismo, el modernismo es un paradigma muy reduccionista, unidimensional, porque enfatiza una única dimensión de la actividad matemática.

El modernismo es un PDR cuyo modelo epistemológico subyacente (MER) es un modelo epistemológico cuasiempirista de las matemáticas -en el sentido de (Lakatos, 1978)-. Según este modelo epistemológico, el núcleo de la actividad matemática tiene lugar en el periodo de desarrollo de las teorías matemáticas, cuando todavía son informales, esto es, antes de ser formalizadas. Se trata de los problemas que provienen de la exploración de las regiones fronterizas de los conceptos, del cuestionamiento de la extensión de estos y de la diferenciación de conceptos anteriormente amalgamados.

La incidencia de este modelo epistemológico en las prácticas escolares tiende a identificar la actividad matemática con la exploración de problemas para los que no se dispone de una técnica de resolución, esto es, con las tareas que se realizan cuando todavía no se sabe gran cosa de la solución: tantear técnicas diversas; aplicar algún resultado conocido; buscar problemas semejantes; formular conjeturas; buscar contraejemplos; o intentar resolver un problema un poco diferente, entre otras (en la línea de las heurísticas de Pólya). En coherencia con lo anterior, el paradigma modernista relaciona los fines de la educación matemática con un cierto tipo de actividad matemática "no rutinaria" y, en última instancia, los identifica con la resolución de problemas "abiertos".

Dado que el modernismo tiende a equiparar el aprendizaje de las matemáticas con el descubrimiento inductivo y autónomo, traslada hacia el aprendizaje

${ }^{13}$ En este trabajo no analizaremos el paradigma tecnicista que constituye una primera reacción al teoricismo. Ante el vacío técnico que provoca el teoricismo, aparece el grito defensivo de "volver a lo básico" encarnado en el tecnicismo que enfatiza los aspectos más rudimentarios del trabajo de la técnica, centrándose esencialmente en las técnicas algorítmicas. 
el centro de gravedad del proceso didáctico, mientras que el teoricismo lo situaba en la enseñanza. Los medios $\left(M_{R}\right)$ que el paradigma modernista propone para alcanzar dichos fines están en la línea del "aprender haciendo" y, en general, de los que proponen algunos de los trabajos que se sitúan en los enfoques por proyectos o por indagación y que se integran dentro del denominado inquiry-based mathematics education (IBME) (Artigue y Blomhøj, 2013) y que comparten, en gran medida, los enfoques por competencias (Gascón, 2011). Esto no significa que identifiquemos el IBM y el enfoque por competencias con el paradigma modernista (que es un paradigma ideal). De hecho, la rápida evolución de dichos enfoques muestra algunos rasgos del paradigma de la modelización matemática que describiremos a continuación, lo cual no hace más que confirmar nuestra tesis de que los paradigmas vigentes en las instituciones escolares son siempre "mestizos".

El modernismo constituye una reacción insuficiente y todavía unidimensional al teoricismo y al tecnicismo, añadiendo nuevas limitaciones en el tipo de actividad matemática que es posible llevar a cabo en las instituciones en las que dicho paradigma es dominante. En particular, una de las restricciones que provoca el modernismo al desarrollo de la actividad matemática consiste en el aislamiento y la descontextualización de los problemas que aborda.

\subsection{El PARADIGMA DE LA MODELIZACIÓN MATEMÁTICA COMO COMPLETACIÓN RELATIVA DE LOS PARADIGMAS UNIDIMENSIONALES}

En la medida que están vigentes en las instituciones escolares, los denominados paradigmas unidimensionales -entre los que se encuentran, además del modernismo, el teoricismo y el tecnicismo- se dificulta enormemente la vida escolar de la modelización matemática como mostraremos a continuación. Estas dificultades se manifiestan en múltiples características de la actividad matemática escolar globalmente considerada. A este respecto, podemos citar: el carácter puntual de las praxeologías matemáticas escolares; la rigidez e incompletitud relativa ${ }^{14}$ de las mismas; la tendencia a la algoritmización de las tareas

\footnotetext{
${ }^{14}$ En (Fonseca, 2004, pp. 181-184) se describen siete indicadores del grado de completitud de una praxeología que sintetizamos a continuación. Diremos que una praxeología local es relativamente completa en la medida que: (1) integra los diferentes tipos de tareas (en lugar de considerarlos aisladamente) y contiene tareas relativas al cuestionamiento tecnológico (esto es, tareas relativas al funcionamiento y la justificación de las técnicas); (2) contiene diferentes técnicas para cada tipo de tareas y criterios para elegir entre ellas; (3) trata
} 
matemáticas; la escasa incidencia del cuestionamiento tecnológico de las técnicas y, en general, del bloque tecnológico-teórico, sobre la práctica matemática; la desarticulación entre las diferentes áreas y sectores de la matemática escolar; el autismo temático (Chevallard, 2002) que comporta el olvido de las posibles razones de ser de una obra, y la autosuficiencia de la matemática escolar que aparece encerrada en sí misma y cuya relación con el resto de disciplinas se reduce a un mero aplicacionismo (Bosch, Fonseca y Gascón, 2004; García, et al., 2006; Barquero, Bosch y Gascón, 2011; Sierra, Bosch y Gascón, 2013; Lucas, 2015). Se conforma así un macrofenómeno didáctico disciplinar que se manifiesta en las enormes restricciones institucionales que dificultan la vida normal y el desarrollo de la modelización matemática (MM) en las instituciones escolares.

Como reacción ante las limitaciones (reales o hipotéticas) originadas por la vigencia de los paradigmas unidimensionales y para soslayar este macrofenómeno $\left(\varphi_{R}\right)$ surge el paradigma didáctico de la modelización matemática (PMM). Se trata de un PDR disciplinar que interpreta "aprender matemáticas" como un proceso de construcción de conocimientos (relativos a un sistema matemático o extramatemático) que se lleva a cabo mediante la construcción de un modelo matemático de dicho sistema. Se trata de un paradigma que conecta funcionalmente el momento exploratorio con el tecnológico-teórico, por lo que completa relativamente el teoricismo y el modernismo (Gascón, 2001, pp.148-151).

Para tomar en consideración el momento del trabajo de la técnica (Chevallard, 1999) y completar así el tecnicismo, el PMM debe integrar los talleres de prácticas matemáticas que son dispositivos didácticos cuya principal función consiste en permitir que viva y se desarrolle con normalidad en las instituciones escolares el citado trabajo de la técnica. Los medios $\left(M_{R}\right)$ o estrategias didácticas que plantea el PMM para vehicular la propuesta curricular que prescribe, se sustentan en los recorridos de estudio e investigación (REI) que son dispositivos didácticos especialmente adecuados para superar las restricciones que inciden sobre la vida institucional de la MM (Barquero, Bosch y Gascón, 2011). Dada la gran flexibilidad de los REl, estos permiten integrar en su seno los talleres de prácticas matemáticas y convertir así la actividad de MM en un trabajo sistemático, paciente, profundizado y a largo plazo (Bosch y Gascón, 1994).

como independientes, en la praxis, los objetos ostensivos que sirven para representar las técnicas y el propio funcionamiento de las técnicas; (4) existen tipos de tareas y técnicas "inversas" de las tareas y técnicas habituales; (5) contiene tipos de tareas conducentes a interpretar el funcionamiento y el resultado de aplicar las técnicas; (6) los elementos tecnológicos inciden de manera significativa sobre la práctica matemática; y (7) existen tareas matemáticas "abiertas" y, particular, tareas de modelización matemática. 
Los REl recuperan explícitamente una posible razón de ser de las obras, integrando su trasfondo problemático en el corazón del proceso de estudio y potenciando las diferentes dialécticas o gestos del estudio (Chevallard, 2002) que permiten describir la dinámica de los REl y, consiguientemente, de la actividad de MM que permite llevar a cabo. En cuanto a la estructura de la actividad de MM se esquematiza en cuatro estadios, sin entrar en detalles ni querer prejuzgar una sucesión temporal lineal entre ellos: (1) delimitación (construcción) del sistema a modelizar; (2) elección de las variables relevantes y elaboración del modelo matemático; (3) trabajo técnico dentro del modelo e interpretación de este trabajo y de sus resultados dentro del sistema; (4) el modelo se independiza mediante la formulación de nuevos problemas que pueden requerir llevar a cabo nuevos procesos de modelización (Gascón, 1992, 1994; Bolea, 2002).

Para interpretar adecuadamente el PMM hay que tener en cuenta la forma como se conceptualiza la actividad de MM en la TAD (Bolea, 2002; García et al., 2006). Partiendo de una concepción institucional de la actividad matemática, la TAD postula que la MM puede describirse en términos del juego entre sistemas y modelos con estructura praxeológica. En concreto, la TAD propone tres caracteres diferenciales de la MM: (a) Incluye la modelización intramatemática como un tipo particular muy importante de MM. Aparece así claramente el carácter recursivo de la actividad de MM y, también, su carácter reflexivo, puesto que en la modelización intramatemática el sistema puede hacer el papel de modelo de su modelo. (b) Postula que toda MM presupone la modelización de una praxeología en su totalidad y no sólo de algunos componentes aislados de la misma. (c) Interpreta la MM como un instrumento de articulación y completación progresiva de la matemática y, en particular, de la matemática escolar. De hecho, la TAD describe los procesos de MM como procesos de reconstrucción de praxeologías matemáticas de complejidad creciente: puntuales, locales, regionales y globales (Chevallard, 2002) que, necesariamente, tienen que estar generados por cuestiones problemáticas que se plantea una comunidad de estudio. Esta forma de conceptualizar la MM es la que justifica identificar toda actividad matemática con una actividad de modelización. En consecuencia, se toma la actividad de MM así redefinida como el modelo epistemológico general de la actividad matemática, como el MER subyacente al PMM. En coherencia con esta forma de interpretar la $\mathrm{MM}$, los fines educativos $\left(\mathrm{F}_{R}\right)$ que propugna el PMM se concretan en que la comunidad de estudio llegue a dominar la actividad de MM de todo tipo de sistemas para trabajar con ellos y responder así a cuestiones problemáticas que surgieron en dichos sistemas. 


\section{DE LOS PARADIGMAS DISCIPLINARES A LOS PARADIGMAS PEDAGÓGICOS DE REFERENCIA}

Para analizar la evolución del paradigma pedagógico vigente en las instituciones escolares, la TAD ha construido dos paradigmas pedagógicos de referencia: el paradigma de la visita de las obras (PVO), que pretende modelizar algunos rasgos de la organización escolar clásica, todavía muy visibles en los sistemas escolares actuales; y el paradigma del cuestionamiento del mundo (PCM), que constituye la propuesta de la TAD como meta u horizonte del cambio educativo hacia el que, supuestamente, evolucionan los sistemas educativos actuales (Chevallard, 2013a y 2013b). Ambos pueden considerarse como casos límites de un tipo de PDR posibles, a nivel pedagógico. Y como en todos los paradigmas didácticos situados en este nivel, el modelo epistemológico subyacente es un modelo epistemológico general, habitualmente implícito, de los conocimientos en juego.

\subsection{DEL TEORICISMO DISCIPLINAR AL PARADIGMA PEDAGÓGICO DE LA VISITA DE LAS OBRAS}

El PVO es un PDR que se sitúa en el nivel pedagógico. Se caracteriza, en primer lugar, por proponer para ser estudiadas obras que no muestran la situación problemática que les dio origen, por lo que aparecen como acabadas y cerradas, impidiendo así todo cuestionamiento de estas. En este paradigma se presentan las obras a estudiar como si fuesen monumentos que tienen valor por sí mismos. En consecuencia, los fines educativos del PVO se reducen esencialmente a que los estudiantes visiten y conozcan ciertas obras dadas de antemano, aunque desconozcan sus posibles razones de ser, esto es, aunque no se planteen las cuestiones a las que dichas obras responden.

En definitiva, el PVO puede considerarse como una posible generalización, a nivel pedagógico, del paradigma teoricista que hemos definido a nivel disciplinar en el caso de las matemáticas. En efecto, al igual que el paradigma teoricista, los medios que propone el PVO para alcanzar los fines que propugna se materializan en una presentación autoritaria de las obras que tiende a silenciar todo tipo de preguntas de los estudiantes sobre las mismas. En definitiva, tiende a reducir el papel de los estudiantes al de simples ejecutantes con poca autonomía y, en los casos límite, al de meros espectadores. 
Como en el caso del teoricismo, el PVO tiende a comprimir el proceso didáctico en el momento del primer encuentro con las obras que se visitan y que se identifica con el momento tecnológico-teórico. Por consiguiente, al igual que en el teoricismo, el resto de los momentos están muy condicionados y hasta subordinados al momento dominante. En este paradigma, las obras se "enseñan" (en el sentido de que se "muestran") al estudiante mediante una presentación discursiva, mediante un relato. Por consiguiente, en las instituciones en las que el PVO está vigente ( $\mathrm{o}$, al menos, es predominante) se producen fenómenos relacionados con la trivialización de la actividad del alumno, que es considerada como subsidiaria, con la falta de autonomía de este y con la citada presentación autoritaria de las obras.

\subsection{De La mOdelizaCión matemÁtica al CUESTIONAMIENTO DEL MUNDO}

Como respuesta al PVO, en muchos trabajos de la TAD se propone como meta el PCM que puede considerarse como una posible generalización, a nivel pedagógico, del PMM definido a nivel disciplinar (en el caso de las matemáticas). En efecto, el PCM se concibe como reacción al estado de cosas, real o hipotético, creado por el PVO en la medida que este sigue estando vigente en las instituciones docentes actuales y continúa originando el fenómeno del monumentalismo que impide una verdadera actividad de estudio e investigación (Chevallard, 2013b). Esta reacción o respuesta del PCM ante el monumentalismo, es análoga a la reacción del PMM ante el estado de cosas originado por los paradigmas clásicos unidimensionales (teoricismo, tecnicismo y modernismo) que, como hemos dicho, dificultan la vida escolar de la actividad de MM.

El PCM propone que la actividad de indagación sea el motor de los procesos de aprendizaje, tal y como se lleva a cabo en las comunidades científicas, lo que no excluye el estudio de obras predeterminadas. Esta indagación se genera siempre en torno a una cuestión problemática y, dado que muy raramente esta puede resolverse directamente con los elementos pertenecientes al sistema en el cual se ha generado, se requiere la creación de diferentes tipos de modelos: conceptuales, analógicos, gráficos, físicos o matemáticos, del sistema en cuestión (o de una parte de este) con el objetivo de reformular y simplificar la cuestión problemática inicial y avanzar hacia una posible respuesta. En consecuencia, el PCM plantea unos fines educativos que marcan una dirección de cambio para superar las consecuencias del monumentalismo: crear un nuevo ethos 
cognitivo, caracterizado por la actitud problematizante y abierto al estudio de nuevos problemas (Chevallard, 2013a) y, paralelamente, instaurar las condiciones para que pueda darse el aprendizaje mediante indagación.

Al igual que el PMM (a nivel disciplinar), los medios que propone el PCM (a nivel pedagógico) para alcanzar dichos fines se materializan en praxeologías didácticas capaces de dar cabida a una modalidad de indagación que se expresa en forma de dialéctica entre cuestiones y respuestas. En ambos casos, cada cual en su nivel, las obras que se proponen para ser estudiadas se presentan típicamente en forma de cuestiones abiertas con gran poder generador de cuestiones derivadas, y los medios que se proponen para construir la respuesta se materializan en los recorridos de estudio e investigación (REI).

\section{PARADIGMAS DIDÁCTICOS ASUMIDOS, DE FACTO, POR LA TAD}

Si analizamos la praxis científica de la TAD, observamos que, hasta el momento, la TAD ha propugnado la emergencia del PCM a nivel pedagógico (Chevallard, 2013a, 2013b). En el nivel disciplinar matemático hemos asumido, de facto, el PMM y, en los niveles subdisciplinares, paradigmas compatibles con el PMM que han sido construidos para responder a fenómenos didácticos específicos siempre relacionados con la MM. Lo anterior significa, en primer lugar, que la TAD ha asumido como propios (al menos implícitamente) los fines que dichos paradigmas propugnan y como modelo de la actividad matemática, el modelo epistemológico que sustenta dichos paradigmas, formulando los fines educativos mediante las nociones que estos proporcionan.

Así, por ejemplo, en (Barquero, Bosch y Gascón, 2011), en coherencia con (García et al., 2006), se sitúa la actividad de MM en el corazón de la actividad matemática, afirmándose que la forma como la TAD reconceptualiza la MM no constituye únicamente un aspecto de las matemáticas, sino que puede considerarse un modelo general de la actividad matemática. Se sitúan así los fines de la educación matemática en que las comunidades de estudio lleven a cabo una genuina actividad de investigación científica que les permita responder a las cuestiones problemáticas que surgen en todo tipo de sistemas (matemáticos o extramatemáticos) mediante la construcción de modelos matemáticos de estos y el trabajo sistemático, a largo plazo, profundizado con dichos modelos. De hecho, la introducción de los REI en la metodología didáctica que propugna la TAD está motivada, en primer lugar, por tratarse de un dispositivo didáctico que, 
en conformidad con el PCM, es capaz de transportar a la escuela una actividad de indagación cercana a la que se lleva a cabo en las comunidades científicas. Y, en segundo lugar, la introducción de los REl está justificada, como hemos dicho, por su capacidad de imponer condiciones muy favorables para potenciar el papel de la MM.

Pero, en sentido estricto, la asunción de un paradigma concreto por parte de una PID contradice claramente nuestra tesis según la cual la ciencia didáctica no está legitimada para hacer juicios de valor de ningún tipo (Gascón y Nicolás, 2016/2019a, 2017, 2018, 2019b). En particular, esta tesis niega la legitimidad de la ciencia didáctica y, por tanto, de los didactas como científicos, para valorar unos fines educativos por encima de otros y, en definitiva, para "preferir" o considerar que un paradigma didáctico es "mejor" o "más valioso" que otro. Como científicos, sólo estamos legitimados para construir PDR y utilizarlos como instrumentos metodológicos (como hipótesis científicas) para estudiar los paradigmas didácticos vigentes a lo largo de la historia o, en general, los paradigmas posibles. Por tanto, en coherencia con dicha tesis, la TAD no tiene legitimidad científica para considerar que, a nivel pedagógico, el PCM es "mejor" o "más valioso" que el PVO. Tampoco tiene legitimidad para preferir el PMM por encima de otros paradigmas disciplinares como el teoricismo, el tecnicismo o el modernismo. Para lo que sí está legitimada la TAD, es para construir y utilizar un PDR como una hipótesis científica, tal como precisaremos a continuación.

Construir un PDR $=\left[M E R, F_{R}, M_{R}, \varphi_{R}\right]$ supone, en primer lugar, postular la coherencia interna del mismo. Esto significa postular que, en cierta institución docente: (a) los medios $M_{R}$ que propone, serán útiles para alcanzar los fines $F_{R}$ que propugna; (b) el modelo epistemológico MER que sustenta el paradigma, permitirá sacar a la luz los fenómenos $\varphi_{R}$ a los que este paradigma pretende responder; y (c) los fines $F_{R}$ se podrán formular con las nociones y los términos que proporciona el MER subyacente. Sin olvidar que, como toda hipótesis científica, la coherencia interna del PDR deberá ser contrastada empíricamente.

Siguiendo esta lógica, interpretaremos los PDR que se han construido y utilizado en las investigaciones llevadas a cabo por la comunidad científica de la TAD como hipótesis científicas. De esta manera los resultados de la investigación didáctica se pueden interpretar en términos de medios-fines. De hecho, siempre es posible interpretar de esta forma los resultados de las citadas investigaciones. Así, por ejemplo, en un trabajo sobre la transposición didáctica de las organizaciones matemáticas, se afirma: 
Dado que las técnicas de modelización están entre las técnicas matemáticas menos visibles, menos "algoritmizables", menos "atomizables" y, en definitiva, más dificilmente evaluables, las restricciones originadas por la necesidad de evaluar la eficacia de los procesos de enseñanza y aprendizaje de las matemáticas, dificulta de manera muy decisiva el proceso de algebrización porque tiende a restringir la utilización sistemática del instrumento algebraico como instrumento de modelización matemática (Bolea, Bosch y Gascón, 2001, p. 294)

Esta afirmación puede interpretarse, sin forzar demasiado las cosas, como formando parte de la problemática ecológica (Gascón y Nicolás, 2016/2019a). En efecto, poniendo entre paréntesis el "valor" que, de manera más o menos explícita, el texto asigna al uso del instrumento algebraico como instrumento de modelización matemática, lo que se está enfatizando es un tipo particular de restricciones transpositivas (derivadas de la necesidad didáctica de evaluar los procesos de estudio) que inciden sobre el desarrollo escolar de dicho instrumento.

Obviamente, no es casual que se indague cuáles son los medios que permitirían alcanzar fines propugnados por el PMM o por un paradigma subdisciplinar coherente con él. Este hecho pone de manifiesto que la elección de los problemas de investigación que hacen las comunidades científicas depende de postulados asumidos por la comunidad de manera más o menos explícita y que, como tales, no pueden ser establecidos racionalmente.

\section{RELACIONES ENTRE LA INVESTIGACIÓN DIDÁCTICA Y LA PRÁCTICA DOCENTE}

Cada PID interpreta la ciencia didáctica basándose en un conjunto de principios o asunciones básicas que se refieren: al tipo de objetos que pueblan el universo didáctico y a las relaciones entre ellos; al ámbito empírico que la didáctica debe tomar en consideración; a la unidad de análisis de los procesos didácticos; al tipo de problemas didácticos que privilegia; a la metodología de investigación que utiliza; etc. En coherencia con estos postulados, cada PID utiliza, en la práctica, un modelo epistemológico del conocimiento en juego y tiende a asumir, de facto, junto a dicho modelo, unos fines educativos que sean coherentes con el mismo, así como ciertos medios presuntamente útiles para alcanzar dichos fines. En consecuencia, la PID saca a la luz hechos didácticos que dicho modelo, junto a los fines y los medios asociados, permiten visualizar. 
Así, y a pesar de que la ciencia didáctica como tal no está legitimada para preferir un paradigma didáctico por encima de otros, toda comunidad didáctica tiende a considerar como propio un paradigma didáctico concreto, esto es, principalmente, un modelo epistemológico de los conocimientos en juego y unos fines educativos coherentes con dicho modelo. Sucede entonces que dicha comunidad suele integrar, como postulados, en el logos de la PID que comparte, los citados componentes del paradigma y, en su praxis científica, privilegia el estudio de aquellos problemas didácticos que pretenden responder a cuestiones relacionadas con las posibles formas de alcanzar los fines educativos que el paradigma en cuestión propugna.

En lo que sigue pretendemos dar una primera respuesta a las cuestiones que han quedado abiertas y que, como hemos indicado en la introducción, podrían formar parte de una potencial tercera etapa del diálogo entre teorías didácticas (o PID). Para ello, mostraremos en qué forma, y mediante qué mecanismos, los paradigmas que asume, de facto, una comunidad de investigadores en didáctica incide simultáneamente sobre su praxis científica y sobre la práctica docente que promueve. Más concretamente, analizaremos brevemente en qué sentido los paradigmas didácticos que una PID asume (en los diferentes niveles de codeterminación) constituyen el lazo de unión entre los fines de la investigación didáctica que llevan a cabo los miembros de la comunidad que comparte dicha PID y los fines de la enseñanza que marcan dichos paradigmas.

En el caso de la TAD, en lo que se refiere a los fines de la investigación, esto es, a los problemas didácticos que privilegia, a los fenómenos didácticos que se propone explicar y a los resultados que considera admisibles, es obvia su dependencia de los paradigmas didácticos que asume y, en especial, del modelo epistemológico subyacente a dichos paradigmas. En efecto, muchos de los problemas didácticos que la TAD formula, aunque explícitamente no siempre se expresen en términos de medios-fines, aluden a los fines que el paradigma asumido propugna -que se expresan con los términos que proporciona el modelo epistemológico subyacente- y a los medios que este propone. En cuanto a los fenómenos didácticos, la TAD se propone explicar aquellos que el paradigma que asume, sustentado en cierto MER específico, permite hacer visibles en cada caso. Algunos ejemplos de problemas didácticos formulados en el ámbito de la TAD nos ayudarán a precisar las cosas:

[...] sous quelles conditions l'algèbre enseignée ne sera plus enseignée comme une

" arithmétique généralisée ", mais apparaîtra comme un instrument nécessaire pour 
l'étude de l'immense champ de problèmes que découvre la technique du patron A/S et qui, à travers certaines variations de cette technique, culmine dans la modélisation algébrique? (Gascón, 1994-95, p. 60).

¿Qué papel pueden desempeñar los "procesos de modelización" [...] para incidir de manera controlada (control científico) sobre el fenómeno del aislamiento de la proporcionalidad respecto al resto de las relaciones funcionales en la Educación Secundaria y, más allá, sobre el fenómeno de la desarticulación de la matemática escolar? (García, 2005, p. 183)

¿Es didácticamente viable, en el actual Sistema de Enseñanza de las Matemáticas, iniciar a los alumnos de la primera etapa de la ESO en el uso del instrumento algebraico? ¿Qué organización matemática puede tomarse como sistema inicial a modelizar? [...] ¿Qué nuevos dispositivos didácticos se requerirán para llevarlo a cabo? (Ruiz-Munzón, 2010, p. 59).

¿Qué tipo de dispositivos didácticos posibilitarían una integración generalizada de la modelización matemática en los sistemas de enseñanza universitarios de las matemáticas para las ciencias experimentales? ¿Qué condiciones se requieren y qué tipos de restricciones institucionales limitan o impiden su desarrollo? (Barquero, Bosch y Gascón, 2011, p. 341).

¿Qué condiciones se requieren y, en particular, qué restricciones dificultan o impiden el desarrollo normal de la modelización funcional en el paso de Secundaria a la Universidad? ¿Qué papel podría jugar el cálculo diferencial elemental en el establecimiento de las citadas condiciones? [...] (Lucas, 2015, p. 108).

En todos estos casos, y en muchos otros, se formulan problemas didácticos aludiendo a los fines de la educación matemática propugnados por el PMM-0 por un paradigma subdisciplinar de este- y a los medios que dicho paradigma propone. Y, aunque de manera latente en algunos casos, el estudio de cada uno de estos problemas está orientado a sacar a la luz y dar cuenta de determinados fenómenos didácticos.

A nivel pedagógico, los fines educativos que la TAD propugna son muy visibles, en coherencia con el paradigma del cuestionamiento del mundo: 
En el nuevo paradigma, el ciudadano debe convertirse en herbartiano, procognitivo y exotérico. ¿Cómo podemos promover esta nueva ciudadanía? Más allá de estar poseídos por la pasión epistemológica que se necesita para ir del camino de la pura ignorancia hacia el del conocimiento adecuado, una condición crucial es, sin duda, el tiempo asignado al estudio y a la indagación en la vida de un adulto. (Chevallard, 2013a, p. 175).

Sin embargo, en muchas investigaciones didácticas, independientemente de la teoría didáctica o PID en el que se sustenten, los fines de la educación matemática que se propugnan tienden a ser relativamente transparentes e incuestionables y que raramente se enuncian como tales fines.

Una muestra muy llamativa de este fenómeno de ocultación de los fines educativos a nivel disciplinar (matemático) se manifiesta en que muchos problemas de investigación en didáctica están centrados en cuestionar los medios, quedando los fines completamente implícitos. Así, es fácil encontrar problemas didácticos formulados en los siguientes términos: "¿Es $M$ un buen medio de enseñanza?" "¿Con el medio M se mejora la enseñanza?". En estas formulaciones, los fines educativos que, presuntamente, se alcanzarían con los citados medios, no sólo quedan implícitos, sino que pueden acabar siendo absolutamente transparentes. Si aceptamos que los fines educativos son postulados, entonces enunciaremos los problemas didácticos en términos de medios-fines, esto es: "¿Es M un medio de enseñanza eficaz para alcanzar el fin F?"

Digamos para concluir que, para profundizar en el diálogo entre diferentes PID, será preciso contrastar los fines educativos y los modelos epistemológicos subyacentes de los paradigmas asumidos por cada una de ellas. En última instancia, será imprescindible contrastar la compatibilidad entre los paradigmas didácticos asumidos, en la práctica, por las diferentes PID. Pero, antes de enfocar el diálogo en estos términos, será preciso discutir si las nociones de praxeología de investigación didáctica (PID) y de paradigma didáctico asumido por una PID son pertinentes o deben sustituirse por otras.

\section{AGRADECIMIENTOS}

Esta investigación se ha financiado por los proyectos I+D+i: RTI2018-101153A-C22 (MCIU/AEI/FEDER, UE) y RTI2018-101153-B-C21 (MCIU/AEI/FEDER, UE). 


\section{REFERENCIAS}

Artigue, M. y Blomhøj, M. (2013). Conceptualizing inquiry-based education in mathematics. ZDM-The International Journal on Mathematics Education, 45, 797-810.

Artigue, M., Bosch, M. y Gascón, J. (2011a). La TAD face au problème de l'interaction entre cadre théoriques en didactique des mathématiques. En M. Bosch, J. Gascón, A. Ruiz Olarría, M. Artaud, A. Bronner, Y. Chevallard, G. Cirade, C. Ladage y M. Larguier, (Eds.), Un panorama de la TAD (pp. 33-55). CRM Documents, vol. 10. Centre de Recerca Matemàtica.

Artigue, M., Bosch, M. y Gascón, J. (2011b). Research praxeologies and networking theories. En M. Pytlak, T. Rowland y E. Swoboda (Eds.), Proceedings of the Seventh Congress of the European Society for Research in Mathematics Education (pp. 2381-2390). University of Rzeszów.

Barquero, B., Bosch, M. y Gascón, J. (2011). Los Recorridos de Estudio e Investigación y la modelización matemática en la enseñanza universitaria de las Ciencias Experimentales. Enseñanza de las ciencias: revista de investigación y experiencias didácticas, 29 (3), 339-352.

Bartolini Bussi, M. G. (2018). Answer to Gascón y Nicolás. For the learning of mathematics, 38(3), 50-53.

Berlín, I. (2017). El poder de las ideas. Página Indómita.

Bolea, P. (2002). El proceso de algebrización de organizaciones matemáticas escolares. (Tesis de doctorado no publicada). Universidad de Zaragoza.

Bolea, P., Bosch, M. y Gascón, J. (2001). La transposición didáctica de organizaciones matemáticas en proceso de algebrización. El caso de la proporcionalidad. Recherches en Didactique des Mathématiques, 21(3), 247-304.

Bosch, M. y Gascón, J. (1994). La integración del momento de la técnica en el proceso de estudio de campos de problemas de matemáticas. Enseñanza de las Ciencias, 12(3), 314-332.

Bosch, M., Fonseca, C. y Gascón, J. (2004). Incompletitud de las organizaciones matemáticas locales en las instituciones escolares. Recherches en Didactique des Mathématiques, 24(2-3), 205-250.

Bosch, M., Gascón, J. y Trigueros, M. (2017). Dialogue between theories interpreted as research praxeologies: the case of APOS and ATD. Educational Studies in Mathematics, 95, 39-52. https://doi.org/10.1007/s10649-016-9734-3.

Castela, C. (2015). Considering theoretical diversity and networking activities in mathematics education from a sociological point of view. In K. Krainer, N. Vondrová (Eds), Proceedings of the 9th Congress of the European Society for Research in 
Mathematics Education pp.2562-2568 https://hal.archives-ouvertes.fr/CERME9-TWG17/hal-01289422v1

Chevallard, Y. (1985). La transposition didactique ; du savoir savant au savoir enseigné, Paris, La Pensée Sauvage ( $2^{\circ}$ ed. 1991).

Chevallard, Y. (1999). L'analyse des pratiques enseignantes en théorie anthropologique du didactique. Recherches en Didactique des Mathématiques, 19 (2), 221-266.

Chevallard Y. (2002). Organiser l'étude. 3. Écologie et régulation. En J. L. Dorier (Ed.), Actes de la Xlème École d'Été de Didactique des Mathématiques (pp. 41-56). La Pensée Sauvage.

Chevallard, Y. (2013a). Enseñar Matemáticas en la Sociedad de Mañana: Alegato a Favor de un Contraparadigma Emergente. REDIMAT-Jounal of Research in Mathematics Education, 2(2), 161-182. https://doi.org/10.4471/redimat.2013.26

Chevallard, Y. (2013b). La enseñanza de la matemática en la encrucijada: por un nuevo pacto civilizacional. Curso impartido en la Universidad Nacional de Córdoba.

Davis, B. (2018). What sort of science is didactics? For the learning of mathematics, 38(3), 44-49.

Diez, J. A. y Ulises Moulines, C. (2016). Fundamentos de Filosofía de La Ciencia. Ariel.

Durkheim, E. (1924/1991). Educació i Sociologia. Eumo Editorial.

Echevarría, J. (1998). Filosofía de la Ciencia. Ediciones Akal.

Fonseca, C. (2004). Discontinuidades matemáticas y didácticas entre la Secundaria y la Universidad. (Tesis de doctorado). Universidad de Vigo.

García, F. J. (2005). La modelización como herramienta de articulación de la matemática escolar. De la proporcionalidad a las relaciones funcionales. (Tesis de doctorado no publicada). Universidad de Jaén.

García, F. J., Gascón, J., Ruiz Higueras, L. y Bosch, M. (2006). Mathematical modelling as a tool for the connection of school mathematics. ZDM-The International Journal on Mathematics Education, 38(3), 226-246.

Gascón, J. (1992). Què s'entén per resolució de problemes de matemàtiques? BIAIX, 2, 10-17. Gascón, J. (1993). Desarrollo del conocimiento matemático y análisis didáctico: Del patrón análisis-síntesis a la génesis del lenguaje algebraico. Recherches en didactique des mathématiques, 13(3), 295-332.

Gascón, J. (1994). El papel de la Resolución de Problemas en la Enseñanza de las Matemáticas. Educación Matemática, 6(3), 37-51.

Gascón, J. (1994-1995). Un nouveau modèle de l'algèbre élémentaire comme alternative à l' "arithmétique généralisée". Petit x, 37, 43-63. 
Gascón, J. (2001). Incidencia del modelo epistemológico de las matemáticas sobre las prácticas docentes. Revista Latinoamericana de Investigación en Matemática Educativa, 4(2), 129-159.

Gascón, J. (2003). From the Cognitive to the Epistemological Programme in the Didactics of Mathematics: Two Incommensurable Scientific Research Programmes? For the Learning of Mathematics, 23(2), 44-55.

Gascón, J. (2011). ¿Qué problema se plantea el enfoque por competencias? Un análisis desde la Teoría Antropológico de lo Didáctico. Recherches en Didactique des Mathématiques, 31(1), 9-50.

Gascón, J. (2014). Los modelos epistemológicos de referencia como instrumentos de emancipación de la didáctica y la historia de las matemáticas. Educación Matemática, (Special Issue: XXV years), 99-123.

Gascón, J. y Nicolás, P. (2016/2019a). Economía, ecología y normatividad en la teoría antropológica de lo didáctico. Educaçāo Matemática Pesquissa, 21(4), 36-52.

Gascón, J. y Nicolás, P. (2017). Can didactics say how to teach? The beginning of a dialogue between the anthropological theory of the didactic and other approaches. For the Learning of Mathematics, 37(3), 26-30.

Gascón, J. y Nicolás, P. (2018). Paradigmas didácticos y reforma curricular: el caso de la teoría antropológica de lo didáctico. En H. Chaachoua y M. Bosch (Eds.), Sixth International Conference on the Anthropological Theory of the Didactic (pp. 88-102). Autrans.

Gascón, J. y Nicolás, P. (2019b). Research ends and teaching ends in the anthropological theory of the didactic. For the learning of mathematics, 39(2), 42-47.

Godino, J. D., Batanero, C. y Font, V. (2019). The Onto-Semiotic Approach: Implications for the prescriptive character of didactics. For the learning of mathematics, 39(1), 38-43.

Koyré, A. (1961/1994). Pensar la ciencia. Paidós.

Lakatos, I. (1971). Historia de la ciencia y sus reconstrucciones racionales. Tecnos.

Lakatos, I. (1978). Pruebas y refutaciones. La lógica del descubrimiento matemático. Alianza.

Lakatos, I. (1981). Matemáticas, ciencia y epistemología. Alianza.

Lerman, S. (2018). Towards subjective truths in mathematics education. For the learning of mathematics, 38(3), 54-56.

Licera, R.M. (2017). Economía y ecología de los números reales en la Enseñanza Secundaria y la Formación del Profesorado (Tesis de doctorado no publicada). Pontificia Universidad Católica de Valparaíso.

Lucas, C. (2015). Una posible razón de ser del cálculo diferencial elemental en el ámbito de la modelización funcional. (Tesis de doctorado no publicada). Universidad de Vigo. 
Mosterín, J. (2008). Lo mejor posible. Racionalidad y acción humana. Alianza Editorial.

Oktaç, A., Trigueros, M. y Romo, A. (2019). APOS Theory: connecting research and teaching. For the learning of mathematics, 39(1), 33-37.

Piaget, J. y García, R. (1981). Psicogénesis e Historia de la Ciencia. Siglo XXI.

Proulx, J. (2018). Prescriptions and proscriptions on mathematics teaching: interesting cases of lost in translation. For the learning of mathematics, 38(3), 56-57.

Radford, L. (2008). Connecting theories in mathematics education: challenges and possibilities. ZDM-The International Journal on Mathematics Education, 40(2), 317-327.

Ruiz-Munzón, N. (2010). La introducción del álgebra elemental y su desarrollo hacia la modelización funcional. (Tesis de doctorado no publicada). Universitat Autònoma de Barcelona.

Sierra, T. A. (2006). Lo matemático en el diseño y análisis de organizaciones didácticas. (Tesis de doctorado no publicada). Universidad Complutense de Madrid

Sierra, T. A., Bosch, M. y Gascón, J. (2013). El cuestionamiento tecnológico-teórico en la actividad matemática. El caso del algoritmo de la multiplicación. Boletim de Educação Matemática, 27(47), 805-828. https://doi.org/10.1590/s0103-636x2013000400006

Staats, S. y Laster, L. A. (2019). About Time. For the learning of mathematics, 39(1), 44-47.

Weber, M. (1917/2010). Por qué no se deben hacer juicios de valor en la sociología y en la economía. Alianza.

JOSEP GASCÓN PÉREZ

Dirección: Campus de la Universidad Autónoma de Barcelona,

Departamento de Matemáticas, Facultad de Ciencias y Biociencias, Edificio C, Carrer dels Til.lers, 08193 Bellaterra (Barcelona),

Teléfono: (0034)935814538 